\title{
Development of a High-Throughput Kinetics Protocol and Application to an aza-Michael Reaction
}

\author{
Xiao Li ${ }^{\dagger, \ddagger}$ and Anna L. Dunn ${ }^{\dagger *}$ \\ † Pharmaceutical Development, GlaxoSmithKline, Collegeville, PA, 19426, United States \\ $\ddagger$ William A. Brookshire Department of Chemical and Biomolecular Engineering, University of \\ Houston, Houston, TX, 77004, United States \\ *Email: anna.1.dunn@gsk.com
}

\section{Supplementary Information}

\section{Table of Contents}

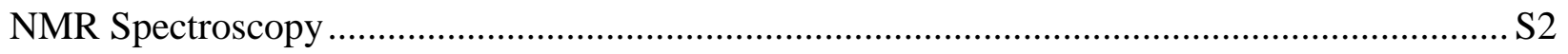

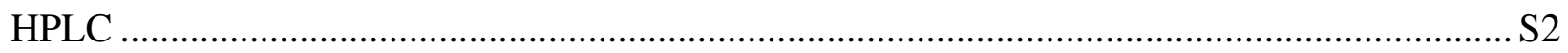

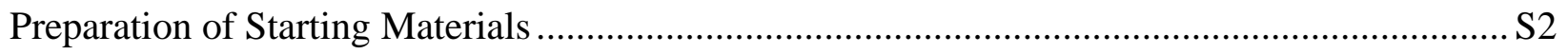

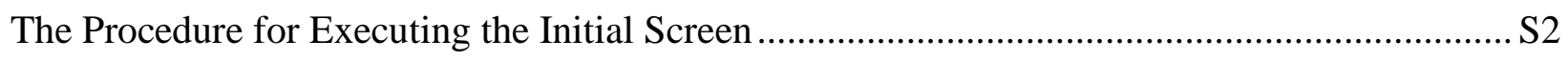

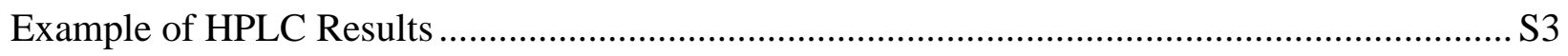

Isolation of Product 3

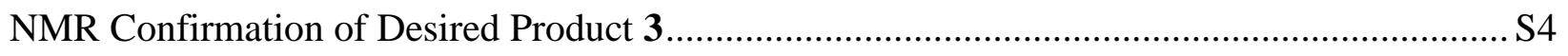

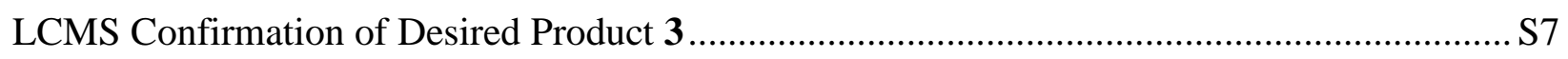

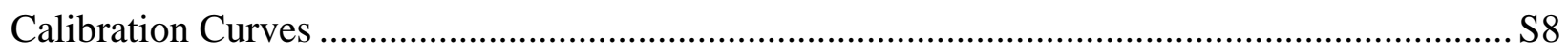

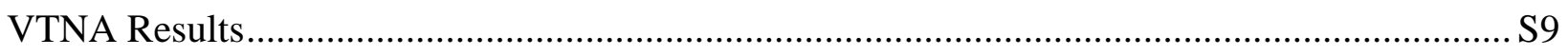

Statistics of Kinetic Model from DynoChem (Mechanism 1) ................................................... S11

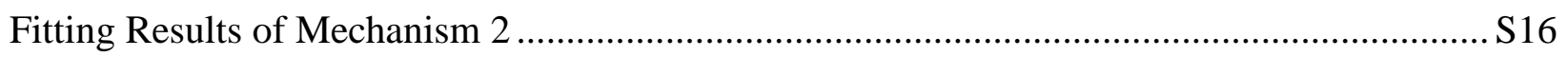




\section{NMR Spectroscopy}

Ambient-temperature NMR spectra were acquired on a Bruker $400 \mathrm{MHz}$ spectrometer. Chemical shifts were calibrated relative to residual protio solvent. Data were processed using MestreNova.

\section{HPLC}

Routine HPLC analysis was performed on Agilent 1290 series instruments. Analysis was completed with traces from a single wavelength with the following method: column, X-select Waters Charged Surface Hybrid $(\mathrm{CSH}) \mathrm{C} 18 \mathrm{XP}, 2.5 \mu \mathrm{m}, 30 \times 2.1 \mathrm{~mm}$; column temperature, 40 ${ }^{\circ} \mathrm{C}$, flow rate, $1 \mathrm{~mL} / \mathrm{min}$ solvent gradient, $\mathrm{H}_{2} \mathrm{O}(0.05 \% \mathrm{TFA} \mathrm{v} / \mathrm{v}) / \mathrm{MeCN}(0.05 \% \mathrm{TFA} \mathrm{v} / \mathrm{v})$, from $97 / 3$ to $5 / 95$ until $3.7 \mathrm{~min}, 5 / 95$ until $4.0 \mathrm{~min}$, from 5/95 to 97/3 until $4.1 \mathrm{~min}, 97 / 3$ until $5.5 \mathrm{~min}$; detection wavelength, $220 \mathrm{~nm}$.

\section{Preparation of Starting Materials}

(a)

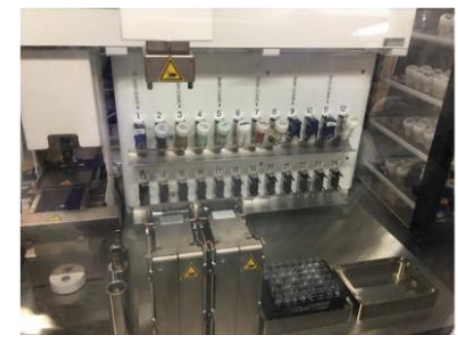

(b)

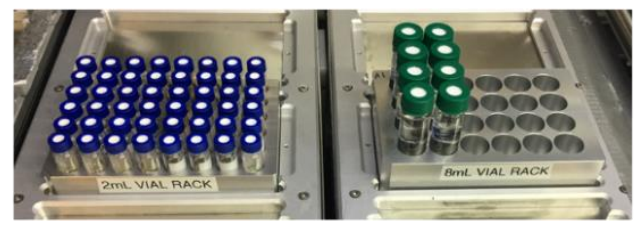

(c)

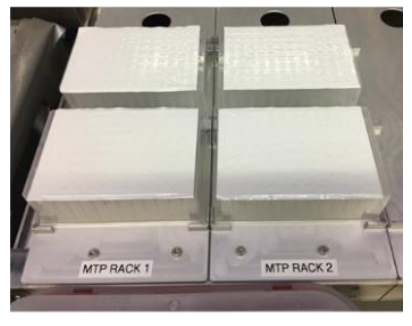

Figure S1. The preparation of (a) reaction vials using QX96 automate solid dosing system, (b) reaction vials and stock solutions, and (c) HPLC vials. To correct for any volumetric sampling errors from the sampling needle or the HPLC auto samplers, area counts are normalized against an external standard (benzonitrile) in the HPLC vials. We found that adding $10 \%$ aqueous $1 \mathrm{M}$ $\mathrm{HCl}$ to the $\mathrm{MeCN}$ in the HPLC vials was effective in stopping any further reactivity, including the reverse reaction.

\section{The Procedure for Executing the Initial Screen}

1. Before sampling, the needlehead lines are equilibrated 10 times to remove any air bubbles, thereby ensuring consistent volume sampling.

2. The tumble stirrer is set at $300 \mathrm{rpm}$ and the temperature control is turned on for $10 \mathrm{~min}$ until the temperature inside the reaction vials is held constant at $25^{\circ} \mathrm{C}$.

3. The automated reaction set-up and quench steps are carried out sequentially. Stock solutions $(0.782 \mathrm{~mL})$ are transferred from $8 \mathrm{~mL}$ vials to the reaction vials, and then samples $(20 \mathrm{uL})$ are transferred from reaction vials to HPLC vials using a 4-NeedleHead liquid handling system at predefined time points and held at room temperature until the following day. Since the effect of reaction time is investigated as a variable, some reactions do not achieve the typical reaction end point. Samples are taken according to the library design until the typical reaction completion time at around $43 \mathrm{~h}$. Time points are recorded in a csv file. 


\section{Example of HPLC Results}

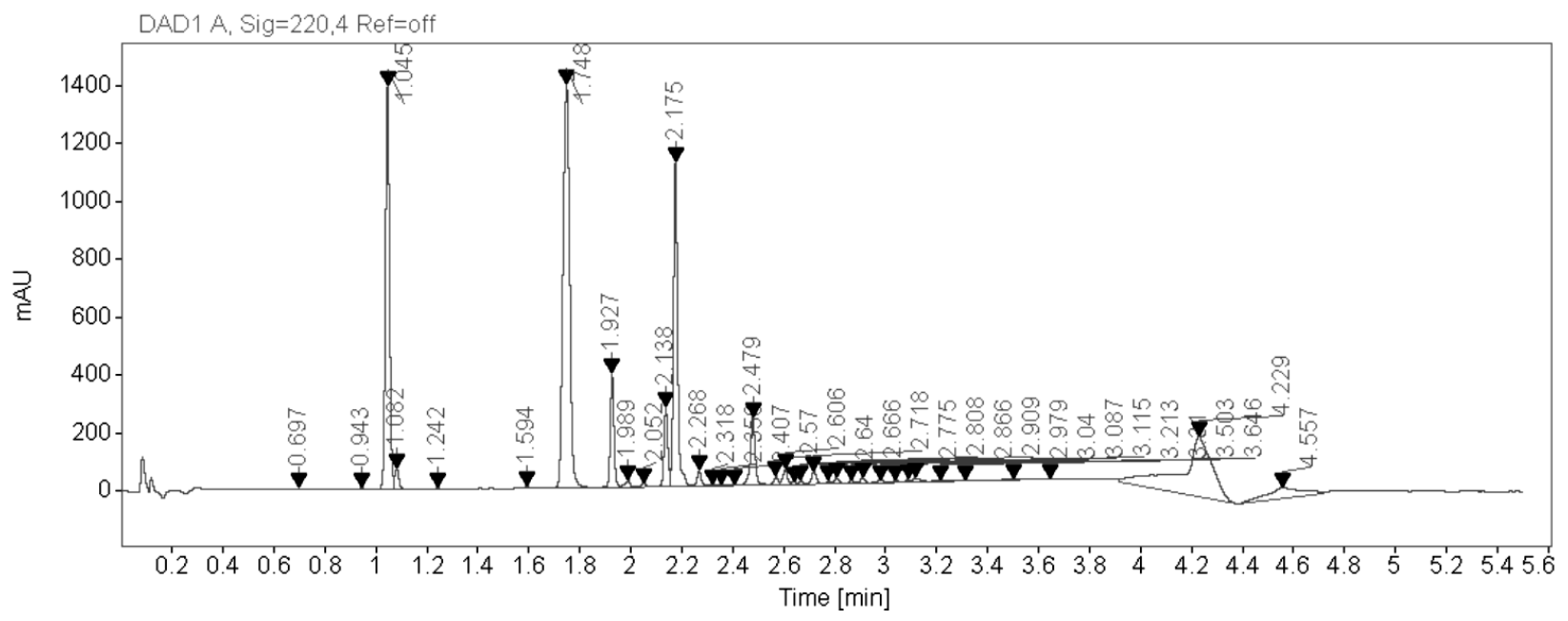

Figure S2. HPLC of aza-Michael reaction of chalcone 1 and ethyl carbamate $\mathbf{2}$ catalyzed by $\mathrm{Fe}(\mathrm{acac})_{3}$ in toluene. Reaction time is $19 \mathrm{~h} 44 \mathrm{~min}$. Peak of product is at $1.927 \mathrm{~min}$. The peak of chalcone 1 is at $2.175 \mathrm{~min}$. The peak of external standard benzonitrile is at $1.045 \mathrm{~min}$.

\section{Isolation of Product 3}

Following literature procedure, ${ }^{31} 36.2 \mathrm{mg} \mathrm{1,} 1.2$ equiv. 2, $10 \mathrm{~mol}_{\%} \mathrm{InCl}_{3}$, and $1 \mathrm{~mL}$ toluene were dosed to an $8 \mathrm{~mL}$ reaction vial. The reaction mixture was stirred at $25^{\circ} \mathrm{C}$ for 18 hours. The resulting product was isolated by washing the organic layer with saturated sodium bicarbonate (3 $\times 5 \mathrm{~mL}$ ), and the solvent was removed via rotatory evaporation followed by isolation by column chromatography (4 g ISCO silica gel column with a linear increase from 0-100\% EtOAc in hexanes). Pure fractions were combined, and solvent was removed via rotatory evaporation. ${ }^{1} \mathrm{H}$, ${ }^{13} \mathrm{C}$, HSQC, COSY, HMBC NMR spectra were acquired on a $400 \mathrm{MHz}$ spectrometer in $\mathrm{CDCl}_{3}$. Spectral elucidation results are displayed below in Figures S3-S7.NMR spectra and LCMS results are consistent with the reported desired product, and key NMR peak assignments are indicated. 
NMR Confirmation of Desired Product 3
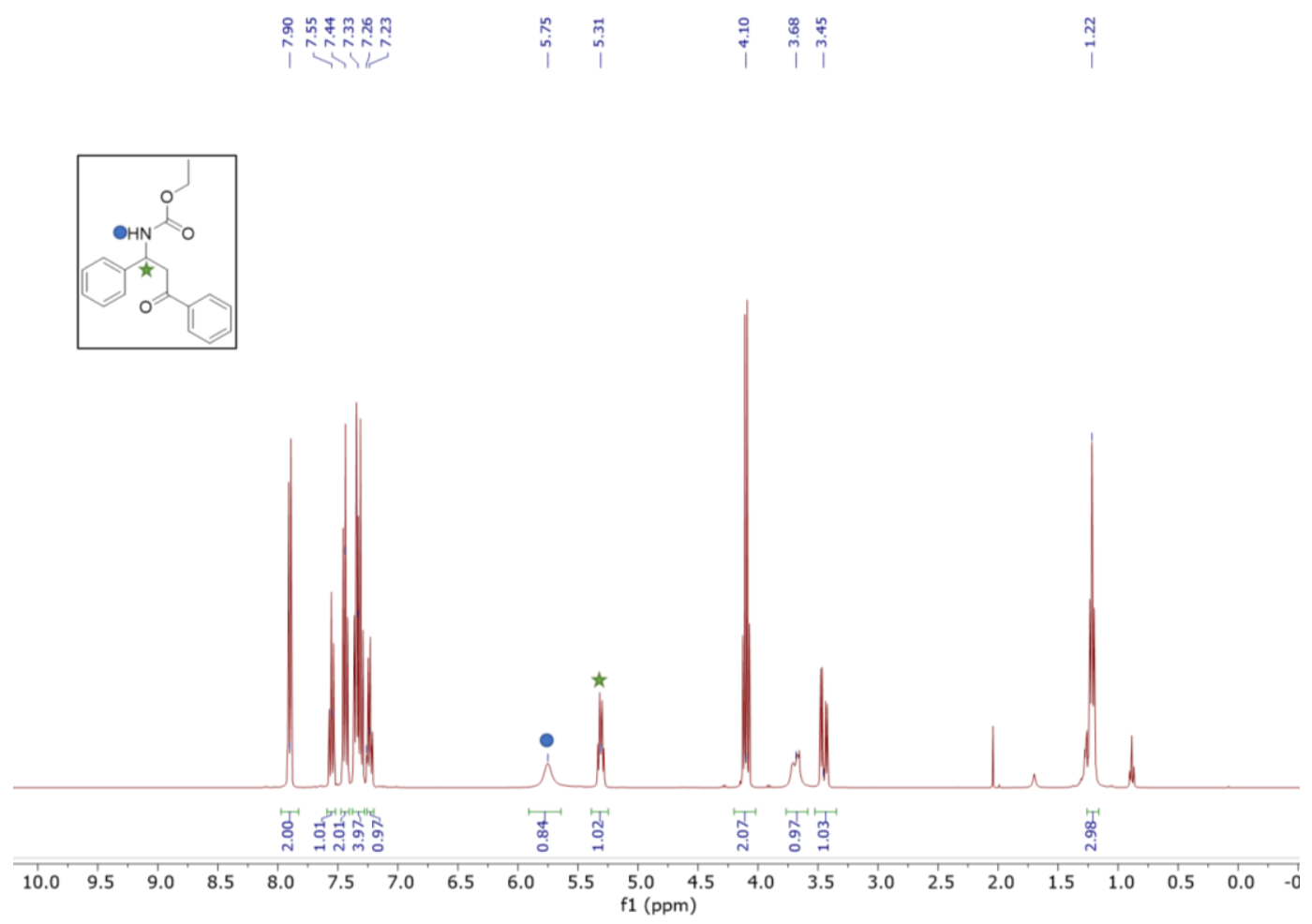

Figure S3. ${ }^{1} \mathrm{H}$ NMR spectrum of product 3. Relevant peak assignments are emphasized.

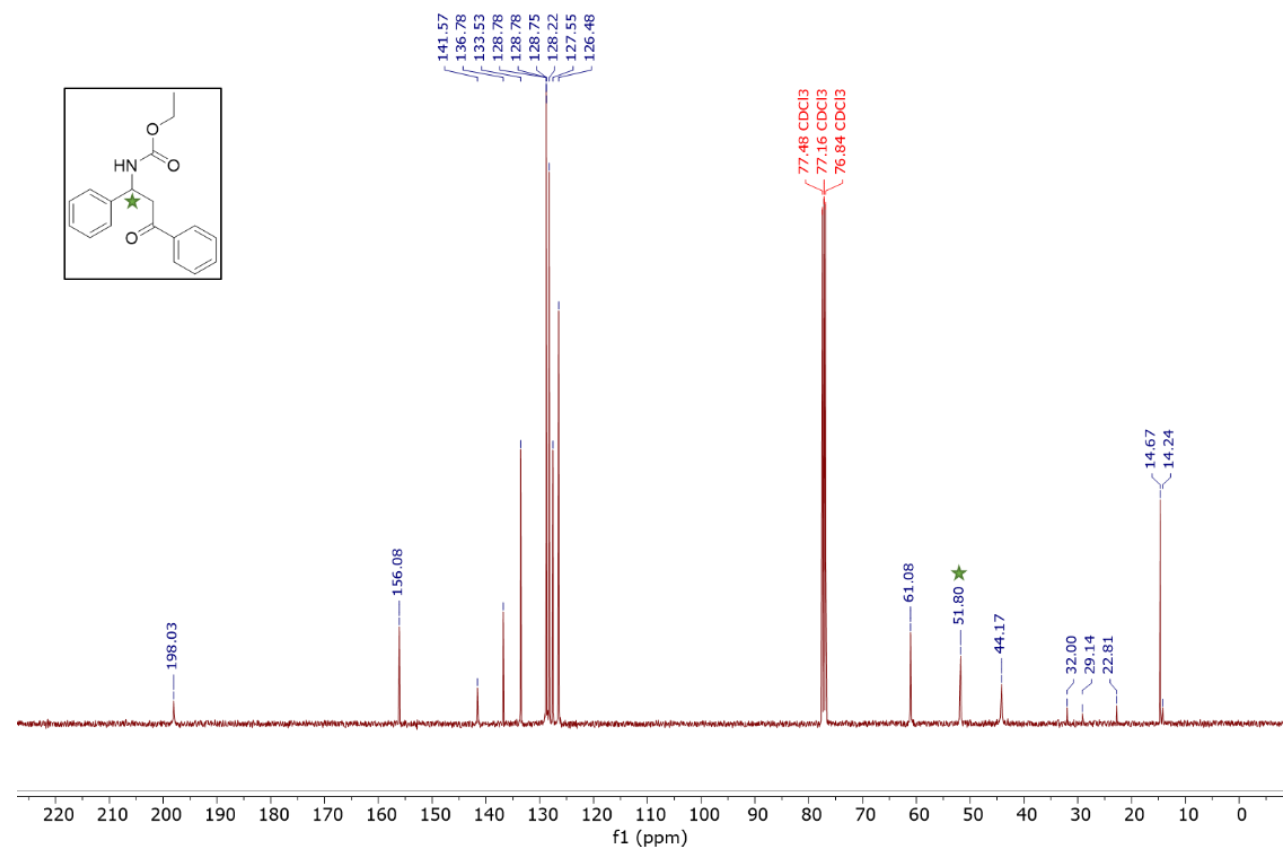

Figure S4. ${ }^{13} \mathrm{C}$ NMR spectrum of product 3. Relevant peak assignment emphasized. 


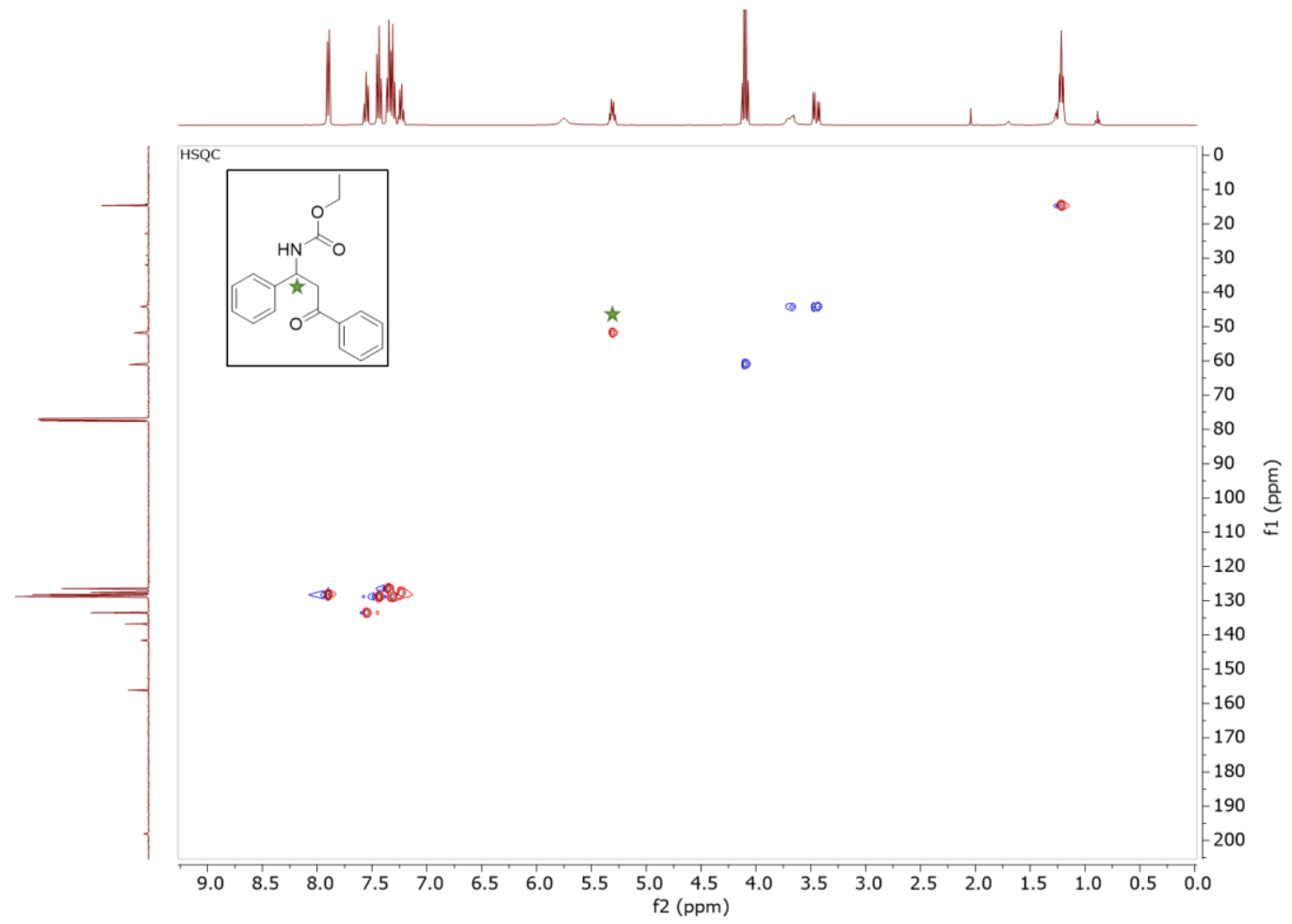

Figure S5. HSQC NMR spectrum of product 3. Relevant peak assignment emphasized.

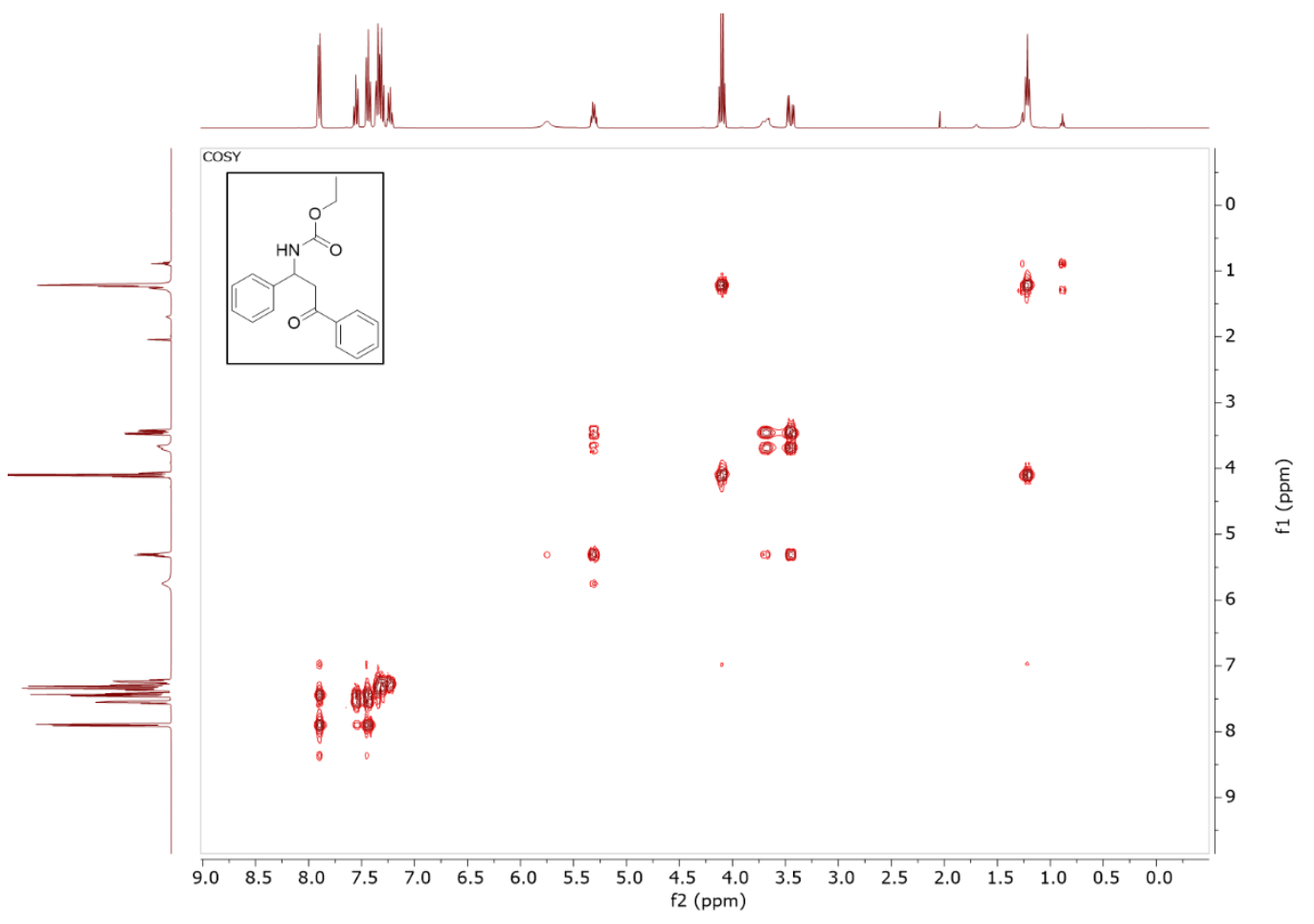

Figure S6. COSY NMR spectrum of product 3. 


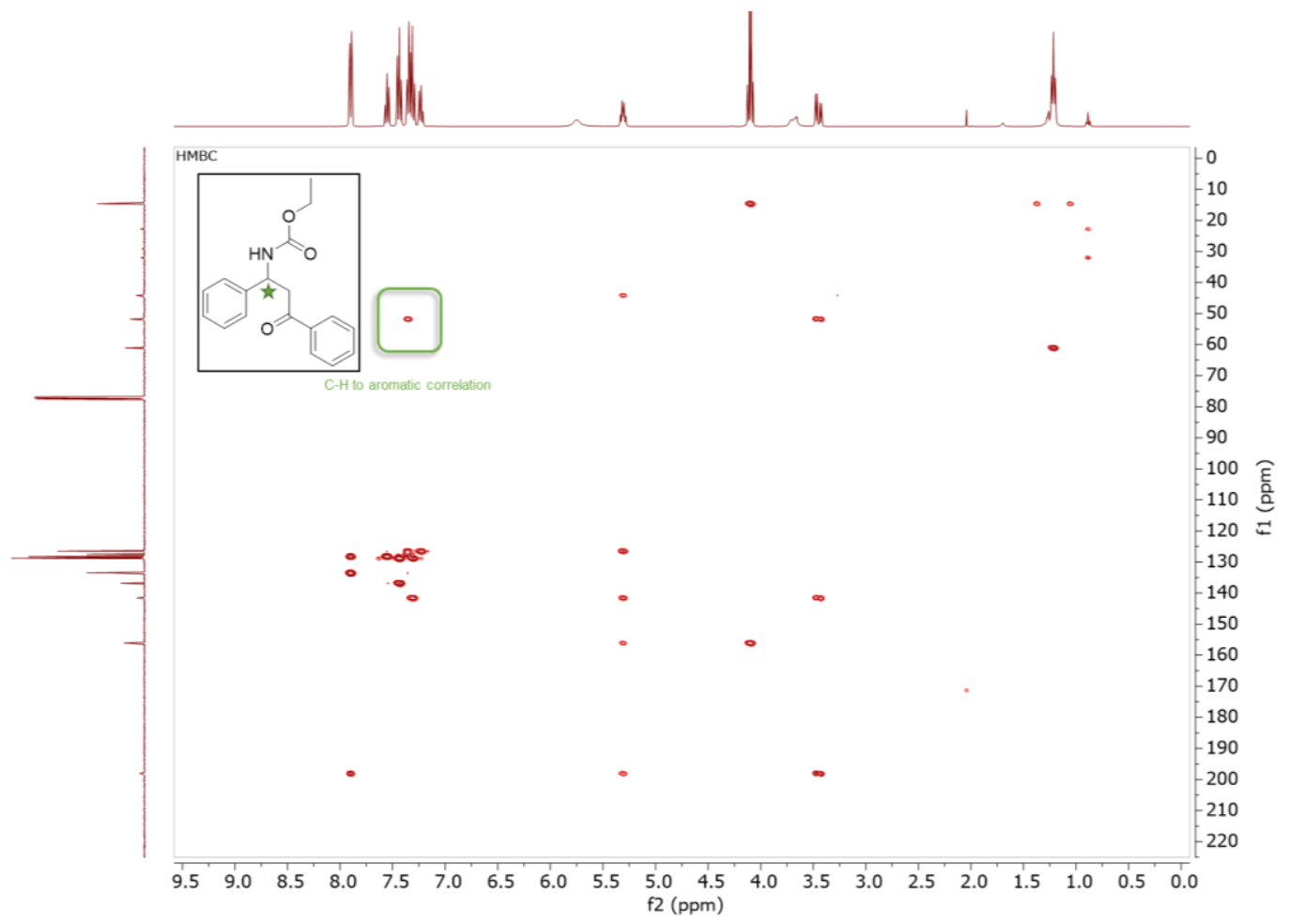

Figure S7. HMBC NMR spectrum of product 3. Relevant peak assignment emphasized. 


\section{LCMS Confirmation of Desired Product 3}

1: MS ES+ :BPI Smooth (SG, 2x1)

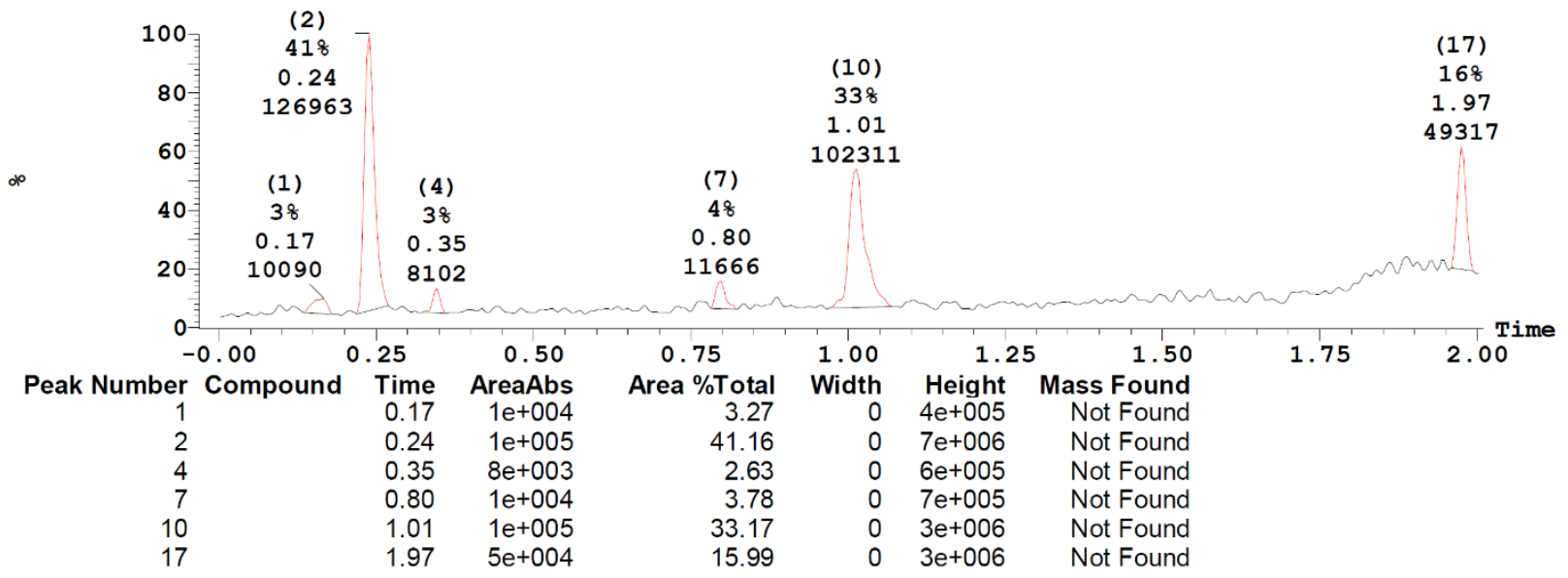

3: UV Detector: TAC: Wavelength Range: (210 - 350)

1. $998 \mathrm{e}+1$

0.16

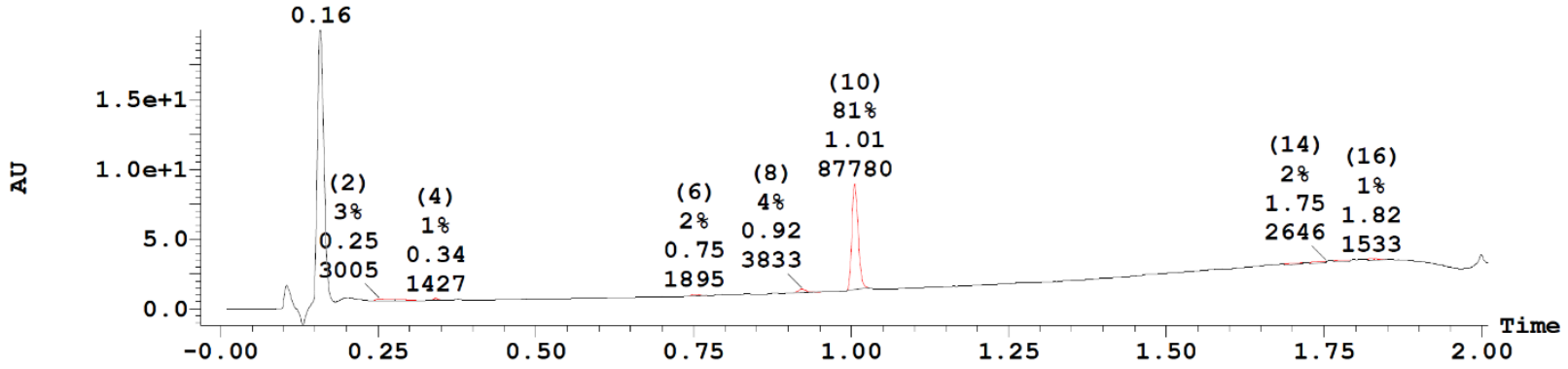

$\begin{array}{rrrrrrr}\text { Peak Number Compound } & \text { Time } & \text { AreaAbs } & \text { Area \%Total } & \text { Width } & \begin{array}{r}\text { Height } \\ \text { Mass Found }\end{array} & \begin{array}{r}\text { Not Found } \\ 2\end{array} \\ 3 & 0.25 & 3 e+003 & 2.76 & 0 & 1 e+005 & \text { Not Found } \\ 4 & 0.28 & 2 e+003 & 1.50 & 0 & 9 e+004 & \text { Not Found } \\ 6 & 0.34 & 1 e+003 & 1.31 & 0 & 1 e+005 & \text { Not Found } \\ 8 & 0.75 & 2 e+003 & 1.74 & 0 & 1 e+005 & \text { Not Found } \\ 10 & 0.92 & 4 e+003 & 3.52 & 0 & 2 e+005 & \text { Not Found } \\ 13 & 1.01 & 9 e+004 & 80.57 & 0 & 8 e+006 & \text { Not Found } \\ 14 & 1.72 & 2 e+003 & 2.19 & 0 & 8 e+004 & \text { Not Found } \\ 15 & 1.75 & 3 e+003 & 2.43 & 0 & 1 e+005 & \text { Not Found } \\ 16 & 1.79 & 3 e+003 & 2.58 & 0 & 1 e+005 & \text { Not Found }\end{array}$

Peak ID Compound Time Mass Found

$10 \quad 1.01 \quad$ Not Found

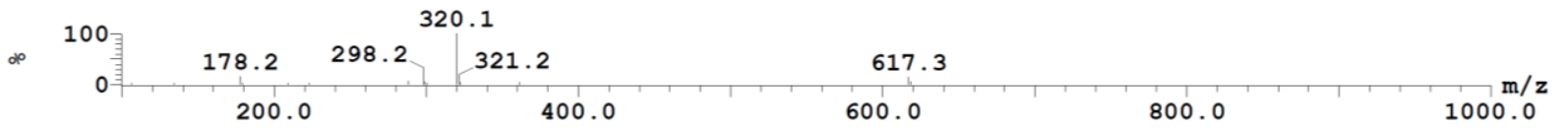

Figure S8. LCMS results of product 3. The molecular weight of $320.1 \mathrm{~g} / \mathrm{mol}$ in peak 10 is the molecular weight of $\mathbf{3}+\mathrm{Na}^{+}$. 


\section{Calibration Curves}

In the case of each calibration for the respective HPLC system and detector, a sample of chalcone 1 and isolated product 3 were each dissolved into $25 \mathrm{~mL}$ volumetric flasks with MeCN. Serial dilutions were performed to make 9 samples and the linear region in the peak area vs. concentration plot was fitted to a straight line.

(a)

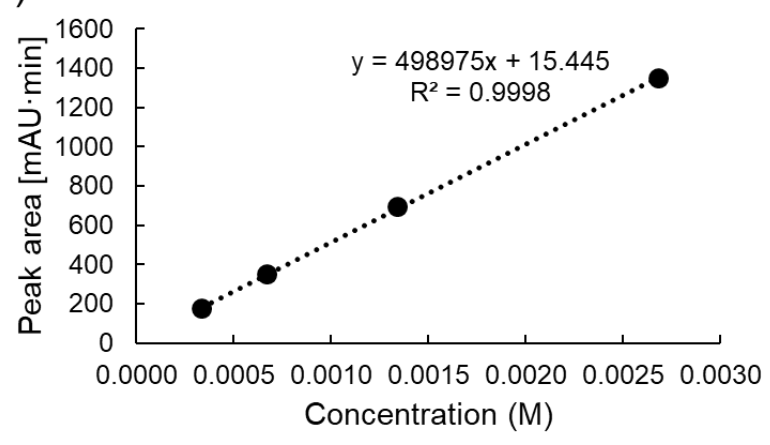

(b)

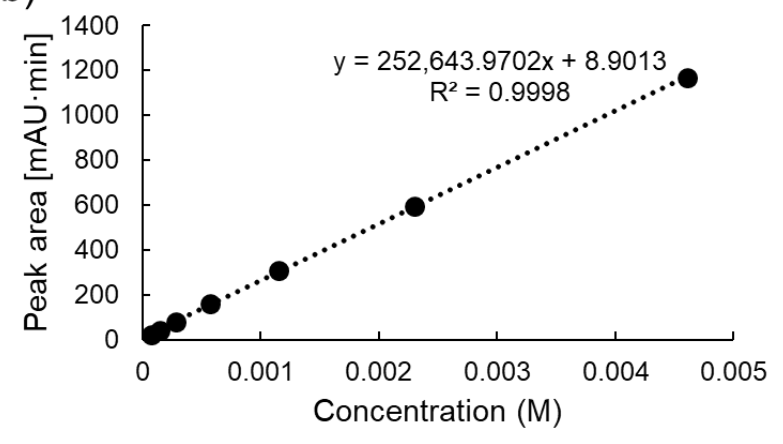

Figure S9. Calibration curves of (a) chalcone 1 and (b) product 3. 


\section{VTNA Results}

(a)

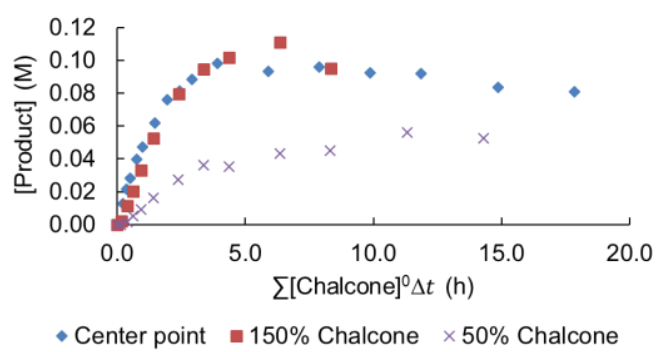

(b)

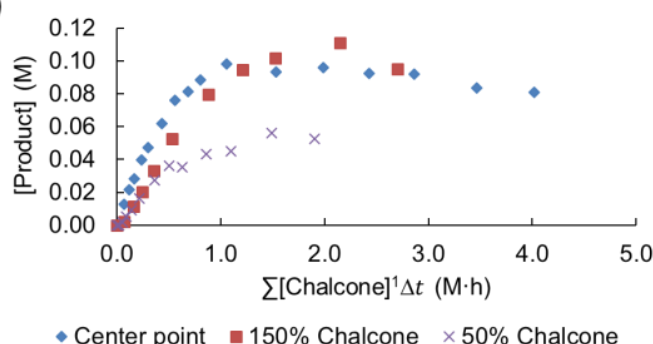

(c)

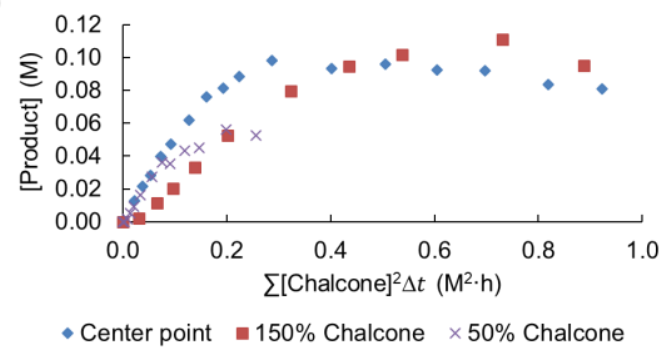

(d)

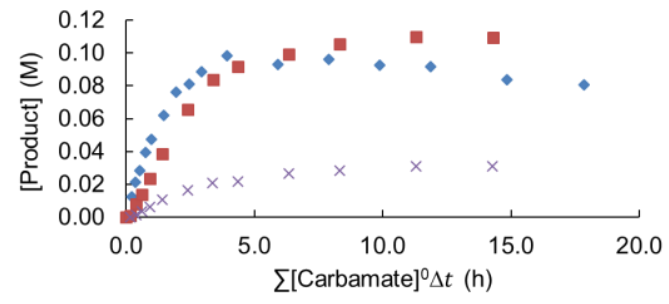

- Center point $=150 \%$ Carbamate $\times 50 \%$ Carbamate

(e)

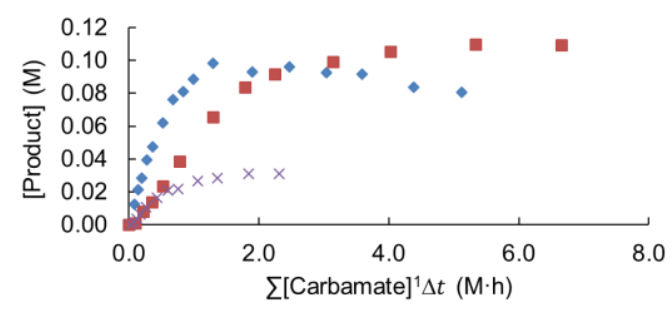

- Center point $=150 \%$ Carbamate $\times 50 \%$ Carbamate

(f)

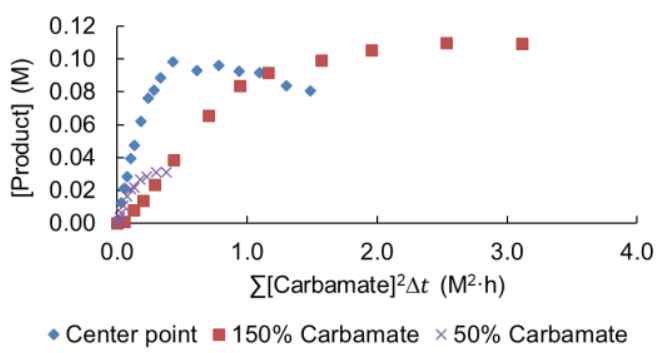

Figure S10. Variable time normalization analysis of (a-c) chalcone 1 [Table 2, entries 1, 8, 9], (d-f) carbamate 2 [Table 2, entries 1-3]. Note that the exponent in the x-axis indicates the order of that reagent. Orders of 0,1 , or 2 are shown for each reagent. Graphical overlay for different experiments indicates successful elucidation of the correct order of that reagent. For a complicated system such as this one, one could argue around which overlay is most correct. This type of analysis does not always provide conclusive results for complicated systems (such as systems which are reversible and display saturation kinetics); however, it gives a quick starting point for what types of kinetic models to begin testing. 
(a)

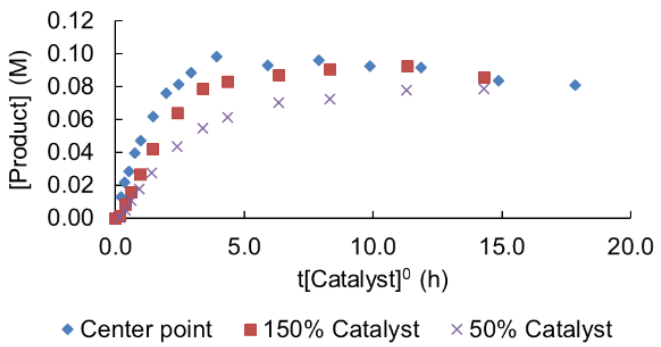

(b)

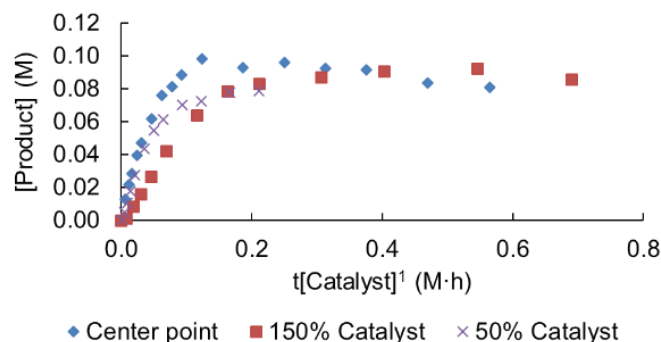

(c)

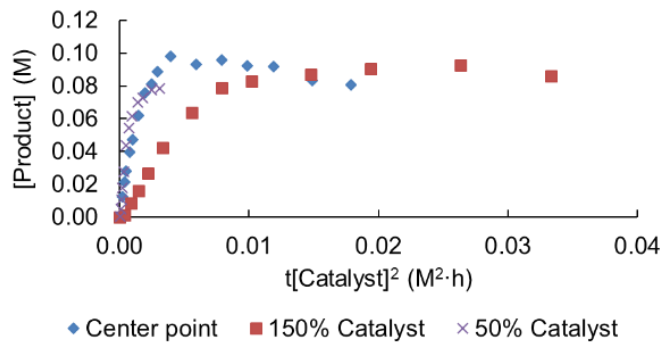

(d)

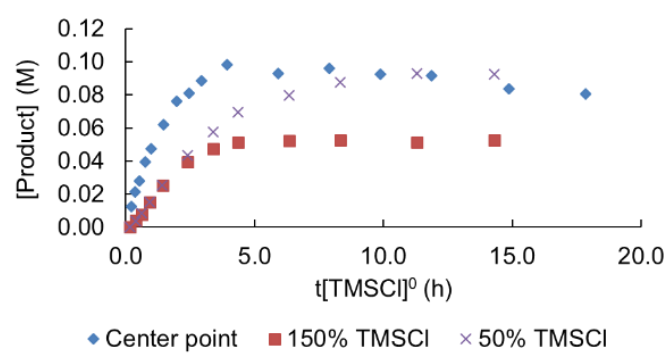

(e)

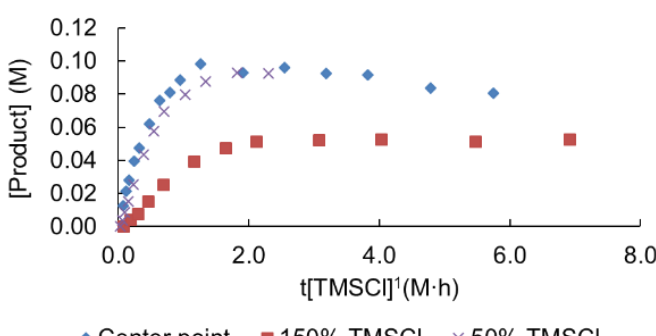

(f)

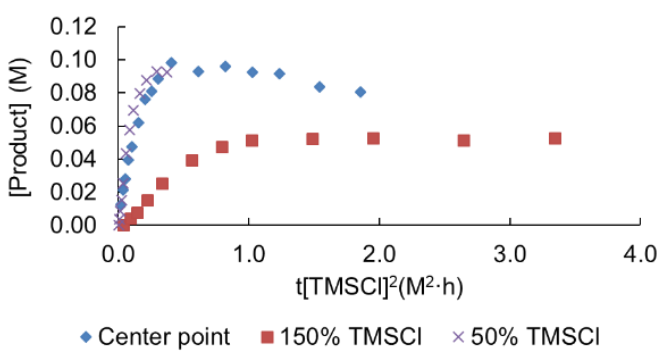

Figure S11. Variable time normalization analysis of (a-c) catalyst $\mathrm{RuCl}_{3}$ [Table 2, entries 1,4 , 5], and (d-f) TMSCl [Table 2, entries 1, 6, 7]. Note that the exponent in the x-axis indicates the order of that reagent. Orders of 0,1 , or 2 are shown for catalyst or TMSCl. Graphical overlay for different experiments indicates successful elucidation of the correct order of catalyst or TMSCl. 


\section{Statistics of Kinetic Model from DynoChem (Mechanism 1)}

The lowest $r^{2}$ value is 0.84 for the product in the experiment with $150 \%$ substrate as show in Table S4. In Figure 6h, data points after 7 hours were removed due to experimental error. The poorest fit (Figure 6j), has additional experimental error from solvent loss, as this was the only run at high temperature.

Table S1. Summary of the Kinetic Model Results simulated in DynoChem using mechanism 1. See Scheme 2 for mechanism.

\begin{tabular}{|c|c|c|c|c|c|c|c|}
\hline Parameters & $\begin{array}{c}\text { Final } \\
\text { Value }\end{array}$ & Units & $\begin{array}{c}\text { Standard } \\
\text { Error }\end{array}$ & $\begin{array}{c}\text { Confidence } \\
\text { Interval }(\%)\end{array}$ & t-Statistic & t crit & Prob (\%) \\
\hline$k_{1, \text { fwd }}$ & $2.65 \mathrm{E}-02$ & $\mathrm{~L}^{2} / \mathrm{mol}^{2} \cdot \mathrm{s}$ & 0.01 & 39.49 & 5.07 & 1.97 & 0.00 \\
\hline$k_{1, \text { rev }}$ & $1.16 \mathrm{E}-01$ & $1 / \mathrm{s}$ & 0.23 & 394.38 & 0.51 & 1.97 & 61.25 \\
\hline$k_{2, \text { fwd }}$ & $1.20 \mathrm{E}-03$ & $\mathrm{~L} / \mathrm{mol} \cdot \mathrm{s}$ & 0.00 & 18.99 & 10.53 & 1.97 & 0.00 \\
\hline$k_{2, \text { rev }}$ & $1.70 \mathrm{E}-03$ & $1 / \mathrm{s}$ & 0.00 & 412.37 & 0.49 & 1.97 & 62.81 \\
\hline$k_{3, \text { fwd }}$ & $1.23 \mathrm{E}+01$ & $\mathrm{~L} / \mathrm{mol} \cdot \mathrm{s}$ & 38.08 & 617.60 & 0.32 & 1.97 & 74.63 \\
\hline$k_{3, \text { rev }}$ & $3.51 \mathrm{E}+01$ & $\mathrm{~L}^{3} / \mathrm{mol}^{3} \cdot \mathrm{s}$ & 82.47 & 469.47 & 0.43 & 1.97 & 67.05 \\
\hline$E_{a 1, \text { fwd }}$ & 41.35 & $\mathrm{~kJ} / \mathrm{mol}$ & 104.21 & 504.08 & 0.40 & 1.97 & 69.19 \\
\hline$E_{a 1, \text { rev }}$ & 80.53 & $\mathrm{~kJ} / \mathrm{mol}$ & 1490.00 & 3700.00 & 0.05 & 1.97 & 95.69 \\
\hline$E_{a 2, f w d}$ & 65.64 & $\mathrm{~kJ} / \mathrm{mol}$ & 34.16 & 104.08 & 1.92 & 1.97 & 5.58 \\
\hline$E_{a 2, \text { rev }}$ & 30.79 & $\mathrm{~kJ} / \mathrm{mol}$ & 1483.00 & 9629.00 & 0.02 & 1.97 & 98.35 \\
\hline$E_{a 3, \text { fwd }}$ & 58.66 & $\mathrm{~kJ} / \mathrm{mol}$ & 2148.00 & 7325.00 & 0.03 & 1.97 & 97.82 \\
\hline$E_{a 3, \text { rev }}$ & 40.11 & $\mathrm{~kJ} / \mathrm{mol}$ & 1553.00 & 7743.00 & 0.03 & 1.97 & 97.94 \\
\hline
\end{tabular}


Table S2. Covariance Matrix from DynoChem for Mechanism 1. See Scheme 2 for mechanism.

\begin{tabular}{|c|c|c|c|c|c|c|c|c|c|c|c|c|}
\hline & $k_{1, f w d}$ & $k_{1, \text { rev }}$ & $k_{2, f w d}$ & $k_{2, \text { rev }}$ & $k_{3, f w d}$ & $k_{3, \mathrm{rev}}$ & $E_{a 1, f w d}$ & $E_{a 1, \text { rev }}$ & $E_{a 2, f w d}$ & $E_{a 2, \mathrm{rev}}$ & $E_{a 3, f w d}$ & $E_{a 3, \text { rev }}$ \\
\hline$k_{1, f w d}$ & 0.00 & 0.00 & 0.00 & 0.00 & -0.12 & -0.32 & 0.04 & 0.01 & 0.02 & 0.11 & -1.02 & -0.98 \\
\hline$k_{1, \text { rev }}$ & 0.00 & 0.05 & 0.00 & 0.00 & 5.10 & -1.21 & -0.24 & 21.01 & -0.23 & 21.07 & 31.75 & 10.39 \\
\hline$k_{2, f w d}$ & 0.00 & 0.00 & 0.00 & 0.00 & 0.00 & 0.00 & 0.00 & 0.00 & 0.00 & 0.00 & -0.04 & -0.04 \\
\hline$k_{2, r e v}$ & 0.00 & 0.00 & 0.00 & 0.00 & 0.07 & -0.02 & 0.01 & 0.32 & 0.00 & 0.35 & 0.35 & 0.04 \\
\hline$k_{3, f w d}$ & -0.12 & 5.10 & 0.00 & 0.07 & 1450.00 & 2419.00 & -204.15 & 2004.00 & -39.36 & 1872.00 & 4903.00 & 2656.00 \\
\hline$k_{3, \text { rev }}$ & -0.32 & -1.21 & 0.00 & -0.02 & 2419.00 & 6801.00 & -461.67 & -640.71 & -6.92 & -843.60 & 2710.00 & 2824.00 \\
\hline$E_{a 1, f w d}$ & 0.04 & -0.24 & 0.00 & 0.01 & -204.15 & -461.67 & 10860.00 & 16360.00 & -1370.00 & 7118.00 & -90060.00 & -94640.00 \\
\hline$E_{a 1, \mathrm{rev}}$ & 0.01 & 21.01 & 0.00 & 0.32 & 2004.00 & -640.71 & 16360.00 & 2219000.00 & -4345.00 & 2201000.00 & 2104000.00 & -97880.00 \\
\hline$E_{a 2, f w d}$ & 0.02 & -0.23 & 0.00 & 0.00 & -39.36 & -6.92 & -1370.00 & -4345.00 & 1167.00 & -1374.00 & -14260.00 & -11030.00 \\
\hline$E_{a 2, \text { rev }}$ & 0.11 & 21.07 & 0.00 & 0.35 & 1872.00 & -843.60 & 7118.00 & 2201000.00 & -1374.00 & 2198000.00 & 2133000.00 & -61190.00 \\
\hline$E_{a 3, f w d}$ & -1.02 & 31.75 & -0.04 & 0.35 & 4903.00 & 2710.00 & -90060.00 & 2104000.00 & -14260.00 & 2133000.00 & 4615000.00 & 2414000.00 \\
\hline$E_{a 3, \text { rev }}$ & -0.98 & 10.39 & -0.04 & 0.04 & 2656.00 & 2824.00 & -94640.00 & -97880.00 & -11030.00 & -61190.00 & 2414000.00 & 2411000.00 \\
\hline
\end{tabular}


Table S3. Correlation Matrix from DynoChem for Mechanism 1. See Scheme 2 for mechanism.

\begin{tabular}{|c|c|c|c|c|c|c|c|c|c|c|c|}
\hline & $k_{1, f w d}$ & $k_{1, \text { rev }}$ & $k_{2, f w d}$ & $k_{2, \text { rev }}$ & $k_{3, f w d}$ & $k_{3, \text { rev }}$ & $E_{a 1, f w d}$ & $E_{a 1, \text { rev }}$ & $E_{a 2, f w d}$ & $E_{a 2, \text { rev }}$ & $E_{a 3, f w d}$ \\
\hline$k_{1, f w d}$ & 1 & 0.06 & 0.58 & 0.22 & -0.6 & -0.74 & 0.07 & 0 & 0.11 & 0.01 & -0.09 \\
\hline$k_{1, \mathrm{rev}}$ & 0.06 & 1 & 0 & 0.95 & 0.58 & -0.06 & -0.01 & 0.06 & -0.03 & 0.06 & 0.06 \\
\hline$k_{2, f w d}$ & 0.58 & 0 & 1 & 0.12 & -0.09 & -0.05 & 0.03 & -0.01 & 0.2 & 0 & -0.17 \\
\hline$k_{2, \text { rev }}$ & 0.22 & 0.95 & 0.12 & 1 & 0.53 & -0.08 & 0.02 & 0.06 & 0.01 & 0.07 & 0.05 \\
\hline$k_{3, f w d}$ & -0.6 & 0.58 & -0.09 & 0.53 & 1 & 0.77 & -0.05 & 0.04 & -0.03 & 0.03 & 0.06 \\
\hline$k_{3, \text { rev }}$ & -0.74 & -0.06 & -0.05 & -0.08 & 0.77 & 1 & -0.05 & -0.01 & 0 & -0.01 & 0.02 \\
\hline$E_{a 1, f w d}$ & 0.07 & -0.01 & 0.03 & 0.02 & -0.05 & -0.05 & 1 & 0.11 & -0.38 & 0.05 & -0.4 \\
\hline$E_{a 1, \text { rev }}$ & 0 & 0.06 & -0.01 & 0.06 & 0.04 & -0.01 & 0.11 & 1 & -0.09 & 1 & 0.66 \\
\hline$E_{a 2, f w d}$ & 0.11 & -0.03 & 0.2 & 0.01 & -0.03 & 0 & -0.38 & -0.09 & 1 & -0.03 & -0.19 \\
\hline$E_{a 2, \text { rev }}$ & 0.01 & 0.06 & 0 & 0.07 & 0.03 & -0.01 & 0.05 & 1 & -0.03 & 1 & 0.67 \\
\hline$E_{a 3, f w d}$ & -0.09 & 0.06 & -0.17 & 0.05 & 0.06 & 0.02 & -0.4 & 0.66 & -0.19 & 0.67 & 1 \\
\hline$E_{a 3, \text { rev }}$ & -0.12 & 0.03 & -0.23 & 0.01 & 0.04 & 0.02 & -0.58 & -0.04 & -0.21 & -0.03 & 0.72 \\
\hline
\end{tabular}


Table S4. Summary of Errors from DynoChem for Mechanism 1. See Scheme 2 for mechanism.

\begin{tabular}{|c|c|c|c|c|c|c|c|}
\hline Scenario name & Data profile name & $\begin{array}{l}\text { Number } \\
\text { of Points }\end{array}$ & $\begin{array}{c}\text { Initial } \\
\text { SSQ }\end{array}$ & $\begin{array}{l}\text { Final } \\
\text { SSQ }\end{array}$ & $\mathrm{rSq}$ & Coeff of Determ & Unweighted SSQ \\
\hline \multirow{2}{*}{$\begin{array}{l}\text { Expt 1: Center point at } \\
\text { reference temperature }\left(40^{\circ} \mathrm{C}\right)\end{array}$} & Solution.Substrate & 16 & 0.02 & 0.03 & 1.00 & 0.90 & $2.80 \mathrm{E}-03$ \\
\hline & Solution.Product & 16 & 0.76 & 0.72 & 0.92 & 0.54 & $6.90 \mathrm{E}-03$ \\
\hline \multirow{2}{*}{$\begin{array}{l}\text { Expt 2: } 1.5 x \text { Reagent at } \\
\text { reference temperature }\left(40^{\circ} \mathrm{C}\right)\end{array}$} & Solution.Substrate & 13 & 0.08 & 0.09 & 0.99 & 0.83 & $6.20 \mathrm{E}-03$ \\
\hline & Solution.Product & 13 & 0.09 & 0.09 & 0.99 & 0.97 & $6.95 \mathrm{E}-04$ \\
\hline \multirow{2}{*}{$\begin{array}{l}\text { Expt 3: } 0.5 x \text { Reagent at } \\
\text { reference temperature }\left(40^{\circ} \mathrm{C}\right)\end{array}$} & Solution.Substrate & 13 & 0.01 & 0.01 & 1.00 & 0.82 & $5.64 \mathrm{E}-04$ \\
\hline & Solution.Product & 13 & 0.29 & 0.32 & 0.96 & 0.89 & $2.02 \mathrm{E}-04$ \\
\hline \multirow{2}{*}{$\begin{array}{l}\text { Expt 4: } 1.5 x \text { Catalyst at } \\
\text { reference temperature }\left(40^{\circ} \mathrm{C}\right)\end{array}$} & Solution.Substrate & 13 & 0.06 & 0.09 & 0.99 & 0.77 & $6.20 \mathrm{E}-03$ \\
\hline & Solution.Product & 13 & 0.20 & 0.18 & 0.98 & 0.94 & $1.00 \mathrm{E}-03$ \\
\hline \multirow{2}{*}{$\begin{array}{l}\text { Expt 5: } 0.5 x \text { Catalyst at } \\
\text { reference temperature }\left(40^{\circ} \mathrm{C}\right)\end{array}$} & Solution.Substrate & 13 & 0.01 & 0.01 & 1.00 & 0.97 & $5.89 \mathrm{E}-04$ \\
\hline & Solution.Product & 13 & 0.15 & 0.18 & 0.98 & 0.93 & $7.47 \mathrm{E}-04$ \\
\hline \multirow{2}{*}{$\begin{array}{l}\text { Expt 6: } 1.5 \mathrm{x} \text { TMSCl at } \\
\text { reference temperature }\left(40^{\circ} \mathrm{C}\right)\end{array}$} & Solution.Substrate & 11 & 0.00 & 0.01 & 1.00 & 0.93 & $6.13 \mathrm{E}-04$ \\
\hline & Solution.Product & 11 & 0.16 & 0.17 & 0.99 & 0.95 & $2.19 \mathrm{E}-04$ \\
\hline \multirow{2}{*}{$\begin{array}{l}\text { Expt 7: } 0.5 \times \mathrm{TMSCl} \text { at } \\
\text { reference temperature }\left(40^{\circ} \mathrm{C}\right)\end{array}$} & Solution.Substrate & 11 & 0.01 & 0.01 & 1.00 & 0.96 & $5.48 \mathrm{E}-04$ \\
\hline & Solution.Product & 11 & 0.08 & 0.08 & 0.99 & 0.97 & $3.36 \mathrm{E}-04$ \\
\hline \multirow{2}{*}{$\begin{array}{l}\text { Expt 8: } 1.5 x \text { Substrate at } \\
\text { reference temperature }\left(40^{\circ} \mathrm{C}\right)\end{array}$} & Solution.Substrate & 10 & 0.05 & 0.07 & 1.00 & 0.71 & 4.10E-03 \\
\hline & Solution.Product & 10 & 1.72 & 1.67 & 0.84 & 0.47 & $8.00 \mathrm{E}-03$ \\
\hline \multirow{2}{*}{$\begin{array}{l}\text { Expt 9: } 0.5 x \text { Substrate at } \\
\text { reference temperature }\left(40^{\circ} \mathrm{C}\right)\end{array}$} & Solution.Substrate & 12 & 0.33 & 0.30 & 0.98 & -0.40 & $4.60 \mathrm{E}-03$ \\
\hline & Solution.Product & 12 & 0.26 & 0.30 & 0.96 & 0.88 & $5.33 \mathrm{E}-04$ \\
\hline \multirow{2}{*}{$\begin{array}{c}\text { Expt 10: Higher temperature } \\
\left(55^{\circ} \mathrm{C}\right)\end{array}$} & Solution.Substrate & 13 & 0.71 & 1.17 & 0.92 & -11.47 & $1.24 \mathrm{E}-01$ \\
\hline & Solution.Product & 13 & 1.95 & 0.30 & 0.97 & 0.88 & $2.50 \mathrm{E}-03$ \\
\hline \multirow{2}{*}{$\begin{array}{l}\text { Expt 11: Lower temperature } \\
\left(25^{\circ} \mathrm{C}\right)\end{array}$} & Solution.Substrate & 10 & 0.38 & 0.14 & 0.99 & 0.66 & $5.80 \mathrm{E}-03$ \\
\hline & Solution.Product & 10 & 2.66 & 0.24 & 0.98 & 0.94 & $9.51 \mathrm{E}-04$ \\
\hline
\end{tabular}


Table S5. Summary of Simulation Parameters from DynoChem for Mechanism 1. See Scheme 2 for mechanism.

\begin{tabular}{|c|c|}
\hline Number of Parameters & 12 \\
\hline Number of data points & 270 \\
\hline Degrees of freedom & 258 \\
\hline SSQ & $\mathbf{6 . 1 7 2}$ \\
\hline Log likelihood, -ln(L) & -693.03 \\
\hline Goodness of Fit & 0.4883 \\
\hline Average Error Value & 0.0242 \\
\hline Notes & No experimental errors were provided. \\
& Average experimental errors have been \\
fitted to be consistent with residuals.
\end{tabular}




\section{Fitting Results of Mechanism 2}

Kinetic modeling was also performed for another potential mechanism, where $\mathrm{RuCl}_{3}$ is involved in both activation steps. An overview of this mechanism, called "Mechanism 2" is outlined in Scheme S1.

Scheme S1. Mechanism 2: Aza-Michael addition via dual activation of chalcone 1 and carbamate 2 by TMSCl and $\mathrm{RuCl}_{3}$.

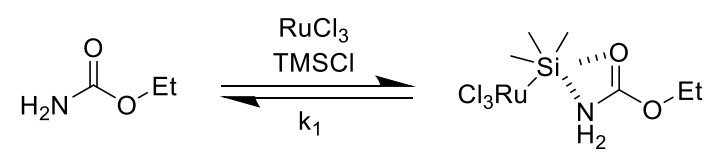

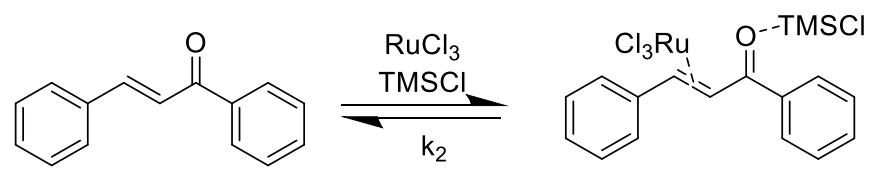<smiles>CCOC(=O)N[C@H](CC(=O)c1ccccc1)c1ccccc1</smiles>

The Dynochem fit for Mechanism 2 is displayed below in Figure S12, and the summary of the modeling results and error analysis are listed in Tables S6-S10. The error analysis revealed that this mechanistic model does not fit the data as well as the model utilizing Mechanism 1. 
(a)

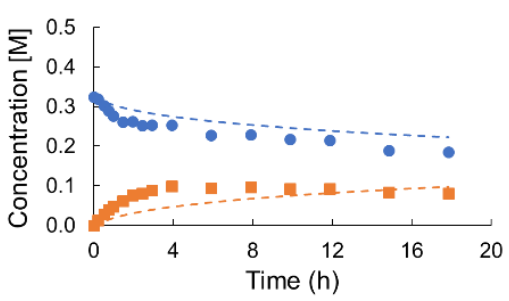

(b)

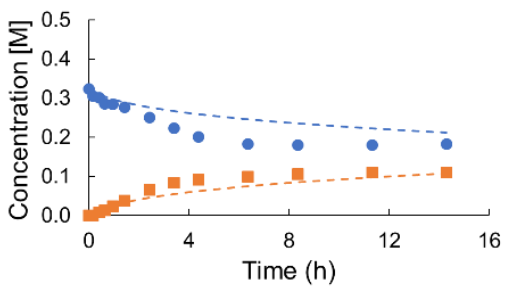

(d)

(c)
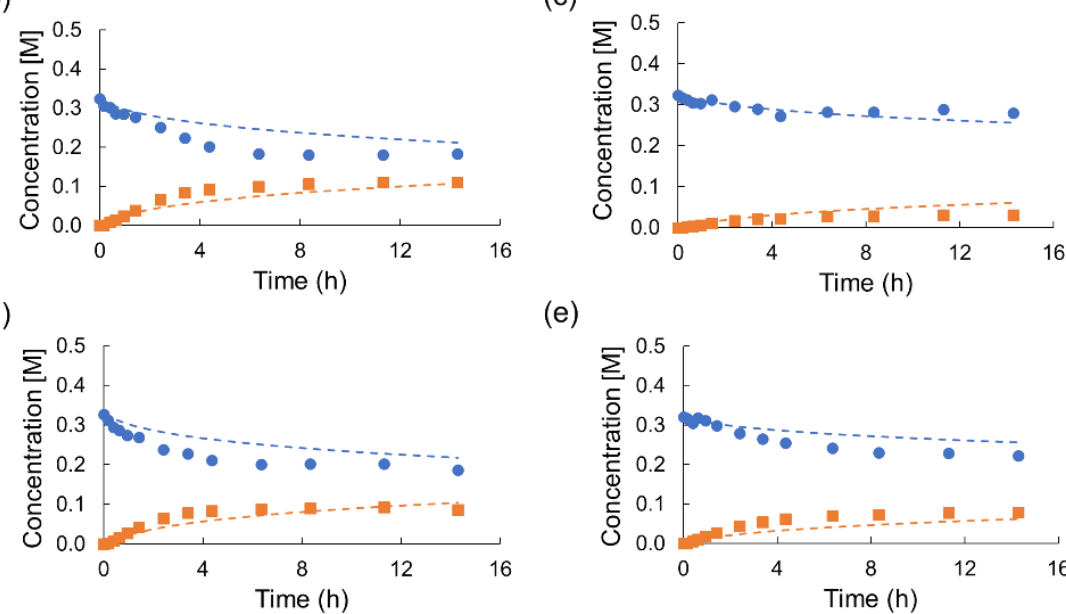

(f)

(e)

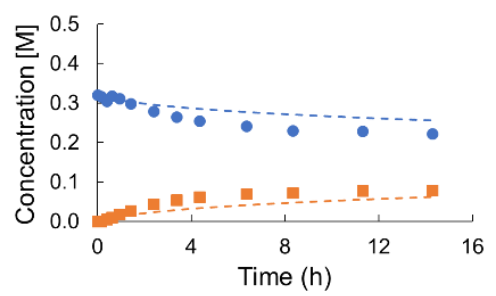

(g)

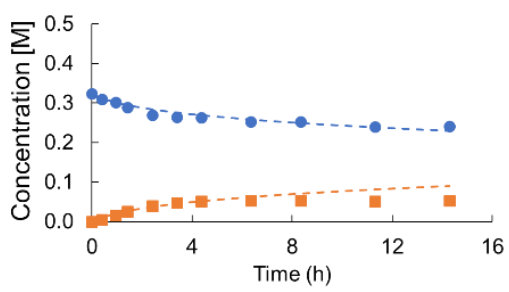

(h)
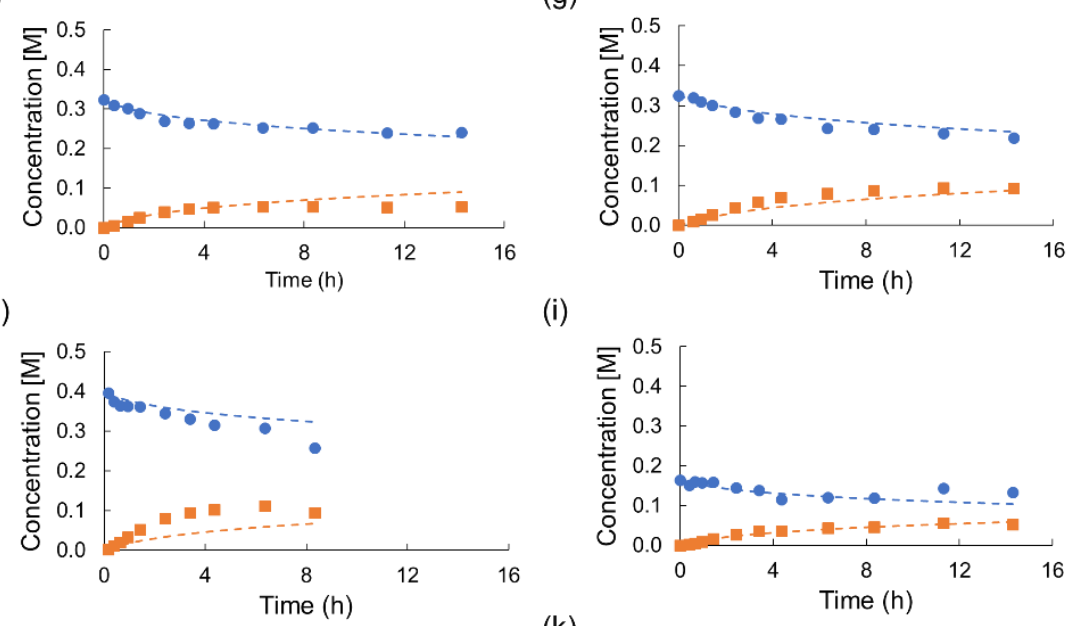

(j)

(i)

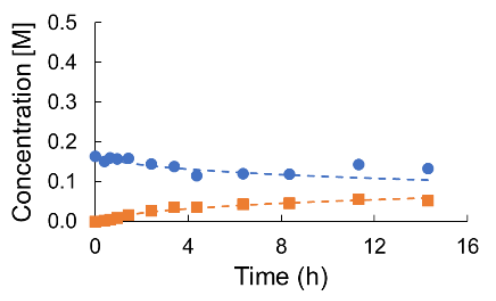

(k)
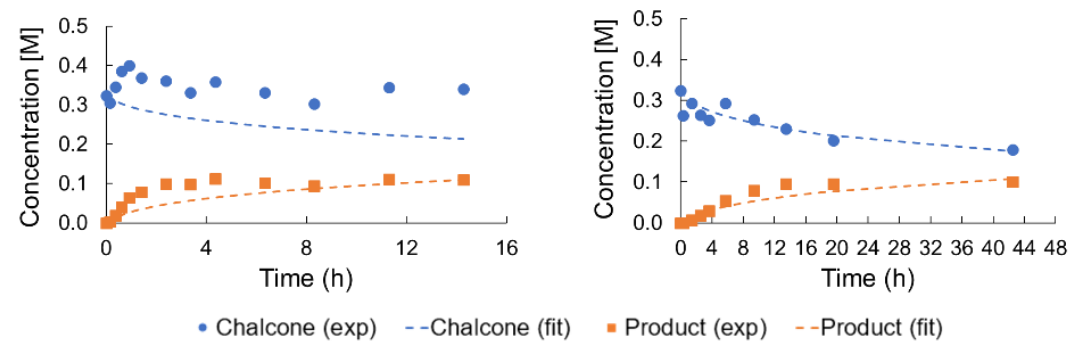

Figure S12. Kinetic profiles for the reactions with (a) Table 1, entry 1, (b) Table 1, entry 2, (c) Table 1, entry 3, (d) Table 1, entry 4, (e) Table 1, entry 5, (f) Table 1, entry 6, (g) Table 1, entry 7, (h) Table 1, entry 8, (i) Table 1, entry 9, (j) Table 1, entry 10, and (k) Table 1, entry 11,. The experiment results of chalcone $\mathbf{1}$ are shown in circles and product $\mathbf{3}$ are shown in squares. The dashed lines are the fitted results from DynoChem using Mechanism 2. 
Table S6. Summary of the Kinetic Model Results simulated in DynoChem using mechanism 2. See Scheme S1 for mechanism.

\begin{tabular}{|c|c|c|c|c|c|c|c|}
\hline Parameters & Final Value & Units & Standard Error & Confidence Interval (\%) & $\mathrm{t}$-Statistic & $\mathrm{t}$ crit & Prob (\%) \\
\hline$k_{1, f w d}$ & $1.08 \mathrm{E}+01$ & $\mathrm{~L}^{2} / \mathrm{mol}^{2} \cdot \mathrm{s}$ & 1511 & 27930 & 0.0072 & 1.969 & 99.429 \\
\hline$k_{1, \text { rev }}$ & $1.29 \mathrm{E}+02$ & $1 / \mathrm{s}$ & 17380 & 26890 & 0.0074 & 1.969 & 99.407 \\
\hline$k_{2, f w d}$ & $1.03 \mathrm{E}-02$ & $\mathrm{~L}^{2} / \mathrm{mol}^{2} \cdot \mathrm{s}$ & 0.0067 & 129.843 & 1.54 & 1.969 & 12.47 \\
\hline$k_{2, \text { rev }}$ & $4.80 \mathrm{E}-03$ & $1 / \mathrm{s}$ & 0.2453 & 10180 & 0.0197 & 1.969 & 98.433 \\
\hline$k_{3, f w d}$ & $5.44 \mathrm{E}+01$ & $\mathrm{~L} / \mathrm{mol} \cdot \mathrm{s}$ & 2776 & 10210 & 0.0196 & 1.969 & 98.438 \\
\hline$k_{3, \text { rev }}$ & $1.20 \mathrm{E}+03$ & $\mathrm{~L}^{4} / \mathrm{mol}^{4} \cdot \mathrm{s}$ & 2431 & 403.732 & 0.4954 & 1.969 & 62.075 \\
\hline$E_{a 1, f w d}$ & 21.83 & $\mathrm{~kJ} / \mathrm{mol}$ & 46620 & 427200 & 0.0004682 & 1.969 & 99.963 \\
\hline$E_{a 1, \text { rev }}$ & 99.14 & $\mathrm{~kJ} / \mathrm{mol}$ & 46620 & 94060 & 0.0021 & 1.969 & 99.831 \\
\hline$E_{a 2, f w d}$ & 41.41 & $\mathrm{~kJ} / \mathrm{mol}$ & 171.061 & 826.143 & 0.2421 & 1.969 & 80.89 \\
\hline$E_{a 2, \text { rev }}$ & 0.00 & $\mathrm{~kJ} / \mathrm{mol}$ & 0 & At Min & 0 & 1.969 & 0 \\
\hline$E_{a 3, \text { fwd }}$ & 111.15 & $\mathrm{~kJ} / \mathrm{mol}$ & 3450 & 6207 & 0.0322 & 1.969 & 97.432 \\
\hline$E_{a 3, \text { rev }}$ & 47.44 & $\mathrm{~kJ} / \mathrm{mol}$ & 4074 & 17170 & 0.0116 & 1.969 & 99.072 \\
\hline
\end{tabular}

Table S7. Covariance Matrix from DynoChem for Mechanism 2. See Scheme S1 for mechanism.

\begin{tabular}{|c|c|c|c|c|c|c|c|c|c|c|c|c|}
\hline & $k_{1, f w d}$ & $k_{1, \text { rev }}$ & $k_{2, f w d}$ & $k_{2, \mathrm{rev}}$ & $k_{3, f w d}$ & $k_{3, \text { rev }}$ & $E_{a 1, f w d}$ & $E_{a 1, r e v}$ & $E_{a 2, f w d}$ & $E_{a 2, r e v}$ & $E_{a 3, f w d}$ & $E_{a 3, r e v}$ \\
\hline$k_{1, f w d}$ & $2.3 \mathrm{E}+6$ & $2.4 \mathrm{E}+7$ & $3.9 \mathrm{E}+0$ & $-1.0 \mathrm{E}+2$ & $-1.3 \mathrm{E}+6$ & $-1.5 \mathrm{E}+6$ & $3.5 \mathrm{E}+7$ & $3.5 \mathrm{E}+7$ & $1.3 \mathrm{E}+4$ & $\mathrm{n} / \mathrm{a}$ & $-3.1 E+5$ & $-2.8 \mathrm{E}+5$ \\
\hline$k_{1, \mathrm{rev}}$ & $2.4 \mathrm{E}+7$ & $3.0 \mathrm{E}+8$ & $1.8 \mathrm{E}+1$ & $3.8 \mathrm{E}+2$ & $3.0 \mathrm{E}+6$ & $-2.0 \mathrm{E}+7$ & $3.9 \mathrm{E}+8$ & $3.9 \mathrm{E}+8$ & $2.1 \mathrm{E}+5$ & $\mathrm{n} / \mathrm{a}$ & $-1.0 \mathrm{E}+7$ & $-1.0 \mathrm{E}+7$ \\
\hline$k_{2, f w d}$ & $3.9 \mathrm{E}+0$ & $1.8 \mathrm{E}+1$ & $4.5 \mathrm{E}-5$ & $-1.1 \mathrm{E}-3$ & $-1.2 \mathrm{E}+1$ & $7.7 \mathrm{E}+0$ & $8.8 \mathrm{E}+1$ & $8.8 \mathrm{E}+1$ & $-5.4 \mathrm{E}-2$ & $\mathrm{n} / \mathrm{a}$ & $1.4 \mathrm{E}+0$ & $1.4 \mathrm{E}+0$ \\
\hline$k_{2, \text { rev }}$ & $-1.0 \mathrm{E}+2$ & $3.8 \mathrm{E}+2$ & $-1.1 \mathrm{E}-3$ & $6.0 \mathrm{E}-2$ & $6.8 \mathrm{E}+2$ & $-7.8 \mathrm{E}+1$ & $-1.4 \mathrm{E}+3$ & $-1.4 \mathrm{E}+3$ & $2.0 \mathrm{E}+0$ & $\mathrm{n} / \mathrm{a}$ & $-2.5 \mathrm{E}+2$ & $-2.6 \mathrm{E}+2$ \\
\hline$k_{3, f w d}$ & $-1.3 \mathrm{E}+6$ & $3.0 \mathrm{E}+6$ & $-1.2 \mathrm{E}+1$ & $6.8 \mathrm{E}+2$ & $7.7 \mathrm{E}+6$ & $-7.2 \mathrm{E}+5$ & $-1.7 \mathrm{E}+7$ & $-1.7 \mathrm{E}+7$ & $2.1 \mathrm{E}+4$ & $\mathrm{n} / \mathrm{a}$ & $-2.7 \mathrm{E}+6$ & $-2.9 \mathrm{E}+6$ \\
\hline$k_{3, \text { rev }}$ & $-1.5 \mathrm{E}+6$ & $-2.0 \mathrm{E}+7$ & $7.7 \mathrm{E}+0$ & $-7.8 \mathrm{E}+1$ & $-7.2 \mathrm{E}+5$ & $5.9 \mathrm{E}+6$ & $-1.3 \mathrm{E}+7$ & $-1.3 \mathrm{E}+7$ & $-2.4 \mathrm{E}+4$ & $\mathrm{n} / \mathrm{a}$ & & $1.9 \mathrm{E}+5$ \\
\hline$E_{a 1, f w d}$ & $3.5 \mathrm{E}+7$ & $3.9 \mathrm{E}+8$ & $8.8 \mathrm{E}+1$ & $-1.4 \mathrm{E}+3$ & $-1.7 \mathrm{E}+7$ & $-1.3 \mathrm{E}+7$ & $2.2 \mathrm{E}+9$ & $2.2 \mathrm{E}+9$ & $6.8 \mathrm{E}+5$ & $\mathrm{n} / \mathrm{a}$ & $-3.8 \mathrm{E}+7$ & $-3.9 \mathrm{E}+7$ \\
\hline$E_{a 1, \text { rev }}$ & $3.5 \mathrm{E}+7$ & $3.9 \mathrm{E}+8$ & $8.8 \mathrm{E}+1$ & $-1.4 \mathrm{E}+3$ & $-1.7 \mathrm{E}+7$ & $-1.3 \mathrm{E}+7$ & $2.2 \mathrm{E}+9$ & $2.2 \mathrm{E}+9$ & $6.6 \mathrm{E}+5$ & $\mathrm{n} / \mathrm{a}$ & $-3.9 \mathrm{E}+7$ & $-3.9 \mathrm{E}+7$ \\
\hline$E_{a 2, f w d}$ & $1.3 \mathrm{E}+4$ & $2.1 \mathrm{E}+5$ & $-5.4 \mathrm{E}-2$ & $2.0 \mathrm{E}+0$ & $2.1 \mathrm{E}+4$ & $-2.4 \mathrm{E}+4$ & $6.8 \mathrm{E}+5$ & $6.6 \mathrm{E}+5$ & $2.9 \mathrm{E}+4$ & $\mathrm{n} / \mathrm{a}$ & $4.7 \mathrm{E}+5$ & $5.8 \mathrm{E}+5$ \\
\hline$E_{a 2, \text { rev }}$ & & & & & & & & & & $\mathrm{n} / \mathrm{a}$ & & \\
\hline$E_{a 3, f w d}$ & $-3.1 \mathrm{E}+5$ & $-1.0 \mathrm{E}+7$ & $1.4 \mathrm{E}+0$ & $-2.5 \mathrm{E}+2$ & $-2.7 \mathrm{E}+6$ & $2.2 \mathrm{E}+5$ & $-3.8 \mathrm{E}+7$ & $-3.9 \mathrm{E}+7$ & $4.7 \mathrm{E}+5$ & $\mathrm{n} / \mathrm{a}$ & $1.2 \mathrm{E}+7$ & $1.4 \mathrm{E}+7$ \\
\hline$E_{a 3, \text { rev }}$ & $-2.8 \mathrm{E}+5$ & $-1.0 \mathrm{E}+7$ & $1.4 \mathrm{E}+0$ & $-2.6 \mathrm{E}+2$ & $-2.9 \mathrm{E}+6$ & $1.9 \mathrm{E}+5$ & $-3.9 \mathrm{E}+7$ & $-3.9 \mathrm{E}+7$ & $5.8 \mathrm{E}+5$ & $\mathrm{n} / \mathrm{a}$ & $1.4 \mathrm{E}+7$ & $1.7 \mathrm{E}+7$ \\
\hline
\end{tabular}


Table S8. Correlation Matrix from DynoChem for Mechanism 2. See Scheme S1 for mechanism.

\begin{tabular}{|c|c|c|c|c|c|c|c|c|c|c|c|c|}
\hline & $k_{1, f w d}$ & $k_{1, \text { rev }}$ & $k_{2, f w d}$ & $k_{2, \text { rev }}$ & $k_{3, f w d}$ & $k_{3, \text { rev }}$ & $E_{a 1, f w d}$ & $E_{a 1, \text { rev }}$ & $E_{a 2, f w d}$ & $E_{a 2, \text { rev }}$ & $E_{a 3, f w d}$ & $E_{a 3, \mathrm{rev}}$ \\
\hline$k_{1, f w d}$ & 1 & 0.93 & 0.38 & -0.28 & -0.3 & -0.41 & 0.5 & 0.5 & 0.05 & -0.06 & -0.05 & 1 \\
\hline$k_{1, r e v}$ & 0.93 & 1 & 0.15 & 0.09 & 0.06 & -0.47 & 0.48 & 0.48 & 0.07 & -0.17 & -0.15 & 0.93 \\
\hline$k_{2, f w d}$ & 0.38 & 0.15 & 1 & -0.65 & -0.64 & 0.47 & 0.28 & 0.28 & -0.05 & 0.06 & 0.05 & 0.38 \\
\hline$k_{2, r e v}$ & -0.28 & 0.09 & -0.65 & 1 & 1 & -0.13 & -0.12 & -0.12 & 0.05 & -0.29 & -0.26 & -0.28 \\
\hline$k_{3, f w d}$ & -0.3 & 0.06 & -0.64 & 1 & 1 & -0.11 & -0.13 & -0.13 & 0.04 & -0.28 & -0.26 & -0.3 \\
\hline$k_{3, \text { rev }}$ & -0.41 & -0.47 & 0.47 & -0.13 & -0.11 & 1 & -0.11 & -0.11 & -0.06 & 0.03 & 0.02 & -0.41 \\
\hline$E_{a 1, f w d}$ & 0.5 & 0.48 & 0.28 & -0.12 & -0.13 & -0.11 & 1 & 1 & 0.09 & -0.24 & -0.2 & 0.5 \\
\hline$E_{a 1, \text { rev }}$ & 0.5 & 0.48 & 0.28 & -0.12 & -0.13 & -0.11 & 1 & 1 & 0.08 & -0.24 & -0.21 & 0.5 \\
\hline$E_{a 2, f w d}$ & 0.05 & 0.07 & -0.05 & 0.05 & 0.04 & -0.06 & 0.09 & 0.08 & 1 & 0.79 & 0.83 & 0.05 \\
\hline$E_{a 2, \text { rev }}$ & -0.06 & -0.17 & 0.06 & -0.29 & -0.28 & 0.03 & -0.24 & -0.24 & 0.79 & 1 & 1 & -0.06 \\
\hline$E_{a 3, f w d}$ & -0.05 & -0.15 & 0.05 & -0.26 & -0.26 & 0.02 & -0.2 & -0.21 & 0.83 & 1 & 1 & -0.05 \\
\hline$E_{a 3, \text { rev }}$ & 1 & 0.93 & 0.38 & -0.28 & -0.3 & -0.41 & 0.5 & 0.5 & 0.05 & -0.06 & -0.05 & 1 \\
\hline
\end{tabular}


Table S9. Summary of Errors from DynoChem for Mechanism 2. See Scheme S1 for mechanism.

\begin{tabular}{|c|c|c|c|c|c|c|c|}
\hline Scenario name & Data profile name & $\begin{array}{l}\text { Number } \\
\text { of Points }\end{array}$ & $\begin{array}{c}\text { Initial } \\
\text { SSQ }\end{array}$ & $\begin{array}{l}\text { Final } \\
\text { SSQ }\end{array}$ & $\mathrm{rSq}$ & $\begin{array}{l}\text { Coeff of } \\
\text { Determ }\end{array}$ & $\begin{array}{c}\text { Unweighted } \\
\text { SSQ } \\
\end{array}$ \\
\hline \multirow{2}{*}{$\begin{array}{l}\text { Expt 1: Center point at } \\
\text { reference temperature }\left(40^{\circ} \mathrm{C}\right)\end{array}$} & Solution.Substrate & 16 & 0.0899 & 0.112 & 0.9889 & 0.5584 & $1.2 \mathrm{E}-02$ \\
\hline & Solution.Product & 16 & 1.688 & 1.566 & 0.826 & -0.0011 & $1.5 \mathrm{E}-02$ \\
\hline \multirow{2}{*}{$\begin{array}{l}\text { Expt 2: } 1.5 x \text { Reagent at } \\
\text { reference temperature }\left(40^{\circ} \mathrm{C}\right)\end{array}$} & Solution.Substrate & 13 & 0.223 & 0.2376 & 0.9799 & 0.5387 & $1.6 \mathrm{E}-02$ \\
\hline & Solution.Product & 13 & 0.5175 & 0.4255 & 0.9494 & 0.8575 & $3.4 \mathrm{E}-03$ \\
\hline \multirow{2}{*}{$\begin{array}{l}\text { Expt 3: } 0.5 x \text { Reagent at } \\
\text { reference temperature }\left(40^{\circ} \mathrm{C}\right)\end{array}$} & Solution.Substrate & 13 & 0.0453 & 0.0257 & 0.9985 & 0.4335 & $1.8 \mathrm{E}-03$ \\
\hline & Solution.Product & 13 & 2.938 & 3.287 & 0.5619 & -0.1813 & $2.1 \mathrm{E}-03$ \\
\hline \multirow{2}{*}{$\begin{array}{c}\text { Expt 4: } 1.5 x \text { Catalyst at } \\
\text { reference temperature }\left(40^{\circ} \mathrm{C}\right)\end{array}$} & Solution.Substrate & 13 & 0.169 & 0.2139 & 0.9818 & 0.4458 & $1.5 \mathrm{E}-02$ \\
\hline & & 13 & 0.5198 & 0.4532 & 0.9504 & 451 & $2.6 \mathrm{E}-03$ \\
\hline \multirow{2}{*}{$\begin{array}{c}\text { Expt 5: } 0.5 x \text { Catalyst at } \\
\text { reference temperature }\left(40^{\circ} \mathrm{C}\right)\end{array}$} & Solution.Substrate & 13 & 0.0976 & 0.1088 & 0.9927 & 0.5624 & $7.4 \mathrm{E}-03$ \\
\hline & Solution.Prod & 13 & 1.184 & 1.045 & 0.8682 & 0.6295 & $4.2 \mathrm{E}-03$ \\
\hline \multirow{2}{*}{$\begin{array}{l}\text { Expt 6: } 1.5 \mathrm{x} \text { TMSCl at } \\
\text { reference temperature }\left(40^{\circ} \mathrm{C}\right)\end{array}$} & Solution.Substrate & 11 & 0.0113 & 0.0126 & & & $6.3 \mathrm{E}-04$ \\
\hline & Solution.Product & 11 & 1.779 & 2.135 & 0.8459 & 0.3456 & $2.8 \mathrm{E}-03$ \\
\hline \multirow{2}{*}{$\begin{array}{l}\text { Expt 7: } 0.5 \mathrm{x} \text { TMSCl at } \\
\text { reference temperature }\left(40^{\circ} \mathrm{C}\right)\end{array}$} & Solution.Substrate & 11 & 0.0207 & 0.031 & 0.9982 & 0.8885 & $1.5 \mathrm{E}-03$ \\
\hline & & 11 & 0.7222 & 0.5815 & 0.9437 & 0.8086 & $2.4 \mathrm{E}-03$ \\
\hline \multirow{2}{*}{$\begin{array}{l}\text { Expt 8: } 1.5 x \text { Substrate at } \\
\text { reference temperature }\left(40^{\circ} \mathrm{C}\right)\end{array}$} & Solution.Substrate & 10 & 0.0852 & 0.1194 & 0.9938 & 0.4925 & 7.3E-03 \\
\hline & Solution.Product & 10 & 2.855 & 2.665 & 0.7501 & 562 & $1.3 \mathrm{E}-02$ \\
\hline \multirow{2}{*}{$\begin{array}{c}\text { Expt 9: 0.5x Substrate at } \\
\text { reference temperature }\left(40^{\circ} \mathrm{C}\right)\end{array}$} & Solution.Substrate & 12 & 0.1731 & 0.1677 & 0.9897 & 0.2253 & $2.5 \mathrm{E}-03$ \\
\hline & Solution.Product & 12 & 0.1107 & 0.0874 & 0.9885 & 0.9657 & $1.6 \mathrm{E}-04$ \\
\hline \multirow{2}{*}{$\begin{array}{l}\text { Expt 10: Higher temperature } \\
\left(55^{\circ} \mathrm{C}\right)\end{array}$} & Solution.Substrate & 13 & 0.4897 & 0.848 & 0.9428 & -8.002 & $9.0 \mathrm{E}-02$ \\
\hline & Solution.Product & 13 & 3.525 & 1.311 & 0.8758 & 0.4904 & $1.1 \mathrm{E}-02$ \\
\hline \multirow{2}{*}{$\begin{array}{l}\text { Expt 11: Lower temperature } \\
\left(25^{\circ} \mathrm{C}\right)\end{array}$} & Solution.Substrate & 10 & 0.3248 & 0.1235 & 0.9924 & 0.7008 & $5.1 \mathrm{E}-03$ \\
\hline & Solution.Product & 10 & 0.5417 & 0.583 & 0.9408 & 0.8546 & $2.3 \mathrm{E}-03$ \\
\hline
\end{tabular}


Table S10. Summary of Simulation Parameters from DynoChem for Mechanism 2. See Scheme S1 for mechanism.

\begin{tabular}{|c|c|}
\hline Number of Parameters & 11 \\
\hline Number of data points & 270 \\
\hline Degrees of freedom & 259 \\
\hline SSQ & $\mathbf{1 6 . 1 4 1}$ \\
\hline Log likelihood, -ln(L) & -563.26 \\
\hline Goodness of Fit & 0.4883 \\
\hline Average Error Value & 0.039 \\
\hline Notes & No experimental errors were provided. \\
& Average experimental errors have been \\
fitted to be consistent with residuals.
\end{tabular}


